\title{
Biosafety Concerns Involving Genetically Modified Mosquitoes to Combat Malaria and Dengue in Developing Countries
}

Graciela R. Ostera

Georgetown University Medical Center

Lawrence O. Gostin

Georgetown University Law Center, gostin@law.georgetown.edu

Georgetown Public Law and Legal Theory Research Paper No. 11-28

This paper can be downloaded free of charge from:

https://scholarship.law.georgetown.edu/facpub/614

http://ssrn.com/abstract=1786997

305 JAMA 930-931 (2011)

This open-access article is brought to you by the Georgetown Law Library. Posted with permission of the author.

Follow this and additional works at: https://scholarship.law.georgetown.edu/facpub

Part of the Health Law and Policy Commons 


\section{Biosafety Concerns Involving Genetically Modified Mosquitoes to Combat Malaria and Dengue in Developing Countries}

Graciela R. Ostera, $\mathrm{PhD}$

Lawrence O. Gostin, JD

$\mathrm{T}$ HROUghout HISTORY, MOSQUITOES HAVE BEEN DISease vectors in human settlements in every region. Today, mosquito-transmitted diseases are present mainly in the equatorial belt, posing major risks to half the world's population and causing disease in 700 million individuals annually. Malaria and dengue are the most prevalent mosquito-borne infections, but West Nile virus in the Americas and chikungunya and Japanese encephalitis in Asia and Oceania are rapidly emerging. ${ }^{1}$ Typical areas and conditions for transmission are increasing, attributable to global climatic changes as well as wider dissemination of virulent viral strains. ${ }^{1,2}$

Malaria causes more than 250 million infections and nearly 1 million deaths annually, ${ }^{3}$ with $90 \%$ of fatal cases occurring in infants and children, typically in sub-Saharan Africa. The malaria parasite, a Plasmodium protozoan, is transmitted through the bite of an Anopheles mosquito. ${ }^{3}$ Dengue infection is traditionally present in hyperendemic regions in Southeast Asia, as well as in Africa and Central and South America. Dengue causes 50 million to 100 million cases annually, of which 500000 progress to the lethal form dengue hemorrhagic fever or dengue shock syndrome. ${ }^{4}$ Dengue is caused by a family of viruses transmitted mainly by the Aedes aegypti mosquito and shares a common ancestry with the causative agent of yellow fever. ${ }^{1,4}$

\section{Scientific Innovations in Mosquito Control}

The global response to mosquito-borne infections still relies on low-technology interventions, such as swamp drainage to reduce larval habitats, indoor spraying with residual insecticides, and the use of insecticide-impregnated nets. Traditional interventions, however, pose challenges, such as the increase of insecticide-resistant mosquitoes, environmental concerns with insecticides, and the difficulty in distributing and reliably using bed nets.

Prompted by global health advocates, scientists are considering innovative solutions such as the elimination of global mosquito populations. ${ }^{5}$ However, elimination would be extraordinarily complex, with some 3500 mosquito species in virtually all geographical regions. Even if it were possible, mosquito elimination could be undesirable because only a small proportion of all mosquitoes are human disease vectors, and wide extermination could have serious ecosystem effects.

Given the impracticality of mosquito elimination, scientists are making significant advances in the generation of genetically modified mosquitoes that are unable to transmit disease. Proponents speculate that genetically modified mosquitoes would cause minimal ecological disruption because targeted mosquito species would still occupy their ecological niche, simultaneously preventing the invasive displacement by other species.

Elucidation of the full-length genomic sequences of Anopheles gambiae (the primary vector for malaria in Africa) in 2002 and Aedes aegypti in 2007 provided the basic tools to modify the vector capacity of these organisms. Scientists have recently confirmed the genetic modification of Anopheles stephensi mosquitoes, the main vector of Plasmodium falciparum malaria in the Indian subcontinent. ${ }^{6}$ The goal is to replace the wild mosquito population with transgenic mosquitoes that are refractory vectors of human disease. Researchers plan to spread the modified genetic information using gene drive systems-a mechanism to spread transgenes in the mosquito population at a faster rate than would be expected of mendelian inheritance. This could constitute a significant risk to biodiversity within national borders, as well as to neighboring countries.

In November 2010, the Wellcome Trust announced that the Malaysian government had approved the release of genetically modified sterile male A aegypti mosquitoes to control dengue, a disease for which drugs or vaccines are not available and bed nets are largely ineffective. ${ }^{7}$ Oxitec, the British biotechnology company that developed the transgenic mosquitoes, had already conducted a trial in the Cayman Islands in 2009/2010. Oxitec's "sterile" male mosquitoes pass a lethal genetic element to their offspring, making them unviable. This self-limited strategy is regarded as relatively safe as it is a new form of the sterile insect technique, commonly used in agriculture, with the exception that in this technique insects are rendered sterile by radiation instead of genetic manipulation. Yet another approach uses a strain of the symbiotic bacterium Wolbachia to infect A aegypti mosquitoes, shortening their life span and interfer-

Author Affiliations: Department of Microbiology and Immunology (Dr Ostera) and O'Neill Institute for National and Global Health Law (Mr Gostin), Georgetown University, Washington, DC.

Corresponding Author: Lawrence O. Gostin, JD, Georgetown University Law Center, 600 New Jersey Ave NW, Washington, DC 20001 (gostin@law.georgetown.edu).

(C2011 American Medical Association. All rights reserved. 
ing with their ability to spread dengue infection to humans. This biological control study is scheduled to start in Queensland, Australia, followed by Vietnam, if the trial proves successful. ${ }^{8}$

\section{The Cartagena Protocol}

The Cartagena Protocol on Biosafety to the Convention on Biological Diversity ${ }^{9}$ is an international agreement that ensures safe handling, transport, and use of living modified organisms, with potential adverse effects on biological diversity and taking into account risks to human health. The treaty entered into force in 2003, with 168 countries having signed. The treaty's language oversees international trade of genetically modified agricultural products, rather than genetically modified insects per se.

Clearly, the unique biosafety concerns with the release of genetically modified mosquitoes require specific guidelines, which were provided by the Ad Hoc Technical Expert Group on Risk Assessment and Risk Management to the Conference of the Parties in Nagoya, Japan, in October 2010. ${ }^{10}$ However, the Ad Hoc Technical Expert Group did not address significant technical issues, such as organisms containing gene drive systems to spread the genetic modification into the natural population. Furthermore, the Cartagena Protocol's definition of living modified organisms does not include mosquitoes carrying a symbiotic bacterium, such as Wolbachia, because this does not constitute a modification through "modern biotechnology."

Overall, the Cartagena Protocol is designed to ensure the safe international exchange of living modified organisms as a commodity, intended to resolve European Union/United States trade disputes involving agrobiotechnological products. The international community therefore urgently needs new regulatory pathways for research and deployment of genetically modified arthropods to control disease. The justification for a new treaty is clear-no state, acting by itself, can regulate the transboundary movement of genetically modified mosquitoes once they are released into the natural environment.

\section{Toward a Global Treaty on Genetic or Biological Modifications of Arthropod Vectors}

A new treaty should establish an international process for rigorous examination of scientific evidence, ethical values, and dispassionate review before genetically or biologically modified arthropod vectors are released into the natural environment.

Scientific Evidence and the Precautionary Principle. The treaty body would have to rigorously examine the available scientific evidence and consider the benefits and risks of the alternative models to introduce genetic or biological modifications of mosquitoes, given their differential ecological effects. If the scientific evidence demonstrates significant disease reduction with low ecological risks, the precautionary principle should not impede meaningful benefits for human health. Conversely, if the treaty body finds the human value is unestablished or the ecological risks are high, it should exercise caution.
The Relative Value of Health and the Environment. The international resolution of complex social problems does not lie solely in scientific assessment, but also entails values. Clearly, both human health and ecological concerns are important and interrelated. The treaty body should give great weight to the ability of science to ameliorate serious risks to human health. A long-term perspective is also important because ecological deterioration not only harms the environment, but can also present future risks to human health.

The Least Harmful Alternative. The treaty body should choose the genetic or biological innovations with the lowest ecological risks. For example, Wolbachia symbionts have been extensively used in fruit fly control, so might be regarded as a lower risk method compared with genetically modified mosquitoes carrying gene drive systems intended to propagate "new" genetic information in the wild mosquito population. Overall, genetically or biologically modified arthropods should be released into the environment only as a last resort.

Independent Deliberation in Advance of Release. Importantly, genetically or biologically modified arthropods should be released into the environment only after a full and independent scientific and ethical evaluation. The current practice whereby private companies, researchers, or states make unilateral decisions without transparency and accountability is unacceptable. The benefits and harms of release will accrue not only to those actors, but also to entire regions of the world. Consequently, fair and dispassionate decision making processes, with broad international agreement, are vital.

Ultimately, the international community needs a legal framework that harmonizes environmental safety with the humanitarian responsibility to assist nations under the social and economic burdens of disease.

Conflict of Interest Disclosures: All authors have completed and submitted the ICMJE Form for Disclosure of Potential Conflicts of Interest and none were reported.

Additional Contributions: We thank John Kraemer, JD/MPH, and Benn McGrady, $\mathrm{PhD}$, of the O'Neill Institute at Georgetown University for their contributions to the conceptualization and writing of this article. They were not remunerated for their contributions.

\footnotetext{
REFERENCES

1. Gould EA, Solomon T. Pathogenic flaviviruses. Lancet. 2008;371(9611): 500-509.

2. Alsop Z. Malaria returns to Kenya's highlands as temperatures rise. Lancet. 2007; 370(9591):925-926

3. Taylor SM, Molyneux ME, Simel DL, Meshnick SR, Juliano JJ. Does this patient have malaria? JAMA. 2010;304(18):2048-2056.

4. Webster DP, Farrar J, Rowland-Jones S. Progress towards a dengue vaccine. Lancet Infect Dis. 2009;9(11):678-687.

5. Fang J. Ecology: a world without mosquitoes. Nature. 2010;466(7305): 432-434.

6. Corby-Harris $V$, Drexler A, Watkins de Jong $L$, et al. Activation of Akt signaling reduces the prevalence and intensity of malaria parasite infection and lifespan in Anopheles stephensi mosquitoes. PLoS Pathog. 2010;6(7):e1001003.

7. Enserink $M$. Science and society: GM mosquito trial alarms opponents, strains ties in Gates-funded project. Science. 2010;330(6007):1030-1031.

8. Enserink M. Infectious diseases: Australia to test "mosquito vaccine" against human disease. Science. 2010;330(6010):1460-1461.

9. Secretariat of the Convention on Biological Diversity. Cartagena Protocol on Biosafety to the Convention on Biological Diversity. http://bch.cbd.int/protocol /publications/cartagena-protocol-en.pdf. Accessed January 31, 2011.

10. Marshall JM. The Cartagena Protocol and genetically modified mosquitoes. Nat Biotechnol. 2010;28(9):896-897.
} 